\title{
BIOSSORÇÃO DE CÁDMIO E PRODUÇÃO DE BIOSSURFACTANTES POR FUNGOS FILAMENTOSOS EM FERMENTAÇÃO SUBMERSA
}

\author{
BIOSSORPTION OF CADMIUM AND BIOSURFACTANTS PRODUCTION FOR \\ FILAMENTOUS FUNGI IN SUBMERGED FERMENTATION
}

\author{
Luciane Maria Colla ${ }^{1 *}$, Marcelo Hemkemeier ${ }^{2}$, Anelise Sertoli Lopes Gil ${ }^{3}$, \\ ${ }^{1}$ Professora do Programa de Pós-Graduação em Engenharia - Universidade de Passo Fundo, lmcolla@upf.br \\ ${ }^{2}$ Professor do Curso de Engenharia Ambiental - Universidade de Passo Fundo, marceloh@upf.br \\ ${ }^{3}$ Aluna do Programa de Pós-Graduação em Engenharia - Universidade de Passo Fundo, anelise_gil@yahoo.com.br \\ *Autor para correspondência: Laboratório de Fermentações - Prédio L1- Universidade de Passo Fundo - BR 285 , \\ Bairro São José - Passo Fundo/RS - CEP: 99052-900 - Cx. Postal 611 - Fone: (54) 3316-8193
}

\begin{abstract}
RESUMO
A remoção de metais empregando microrganismos selecionados tem se tornada promissora, já que os mesmos apresentam alta seletividade e taxa de remoção. O presente trabalho teve como objetivo avaliar a remoção de cádmio via bioprocesso submerso por fungos filamentosos. Os microrganismos utilizados foram fungos filamentosos do gênero Aspergillus ssp, identificados por O-3 e O-8, e do gênero Trichoderma sp, identificado como E-19. O meio de cultivo foi composto por $10 \%$ de farelo de trigo, $10 \%$ de solução salina, $4,5 \%$ de extrato de levedura, $2 \%$ de óleo de soja e $10 \mathrm{mg} / \mathrm{L}$ ou $20 \mathrm{mg} / \mathrm{L}$ de cádmio. Os experimentos foram realizados em erlenmeiers a $30{ }^{\circ} \mathrm{C}$ durante $7 \mathrm{~d}$ em agitador orbital. Alíquotas foram coletadas nos tempos inicial e final do bioprocesso para a determinação da remoção do metal. Amostragens nos tempos 0, 1, 2, 35 e $7 \mathrm{~d}$ foram realizadas para determinação da atividade emulsificante dos meios, a qual indica a produção de biossurfactantes pelos microrganismos. Os fungos O-3 e O-8 apresentaram $100 \%$ de remoção do metal adicionado aos meios de cultivo na fermentação submersa, sendo estas remoções superiores que as obtidas por uma espécie de Thrichoderma (46-49\%). Durante os processos de biossorção, foi observada a produção de biossurfactantes pelos microrganismos estudados, demonstrando a importância destes compostos nos processos de remoção de metais de meios líquidos.

Palavras-chave: bioprocesso submerso, fungos, remoção de metal
\end{abstract}

\begin{abstract}
The removal of metals using selected microorganisms has made promising, since they present high selectivity and rate of removal. The aim of this work was to evaluate the removal of cadmium via submerged bioprocess for filamentous fungi. The microorganisms used were filamentous fungi of the genus Aspergillus spp, identified by O-3 and O-8, and of the genus Trichoderma sp, identified as E-19. The culture medium was composed of $10 \%$ wheat bran, $10 \%$ of saline solution with micronutrients, $4.5 \%$ of yeast extract, $2 \%$ of soybean oil and 10 $\mathrm{mg} / \mathrm{L}$ or $20 \mathrm{mg} / \mathrm{L}$ of cadmium. The experiments were performed in erlenmeiers at $30^{\circ} \mathrm{C}$ for $7 \mathrm{~d}$ in a shaker. Aliquots were collected at beginning and end of the bioprocess for the determination of metal removal. Sampling at $0,1,2,3,5$ and $7 \mathrm{~d}$ were performed to determine the emulsifying activity of the media, which indicates the production of biosurfactants by microorganisms. The Aspergillus strains showed $100 \%$ removal of the metal added to the culture medium in submerged fermentation, superior than Thrichoderma (O-3 and O-8) removals (46-49\%). During the process of biosorption, we observed the production of biosurfactants by the microorganisms studied, demonstrating the importance of these compounds in the process of removal of metals from liquid media.
\end{abstract}

Keywords: submerged bioprocess, fungi, metal removal 


\section{INTRODUÇÃO}

A contaminação de águas e solos por metais tóxicos é um grave problema ambiental (DAS et al., 2008). Os metais tóxicos podem provocar problemas de toxicidade aos organismos expostos a eles (KORF et al., 2008), pois, apresentam alto tempo de meia vida, permanecendo ativos no meio ambiente, e por possuírem alta capacidade de acumulação no corpo humano (JIMENEZ et al., 2004). Segundo Freitas e Melnikov (2006), os efeitos da bioacumulação em longo prazo nem sempre são previsíveis, principalmente no caso de compostos como o cromo, que não se decompõem, ou que apresentam baixa degradabilidade, acumulando-se no meio ambiente, podendo atingir a cadeia alimentar (MERLINO et al., 2010).

Dentre os metais pesado o cádmio, é muito utilizado em vários processos industriais, sendo extremamente tóxico em baixas concentrações causando sérios problemas de saúde (GAAD, 1990). O metal cádmio se apresenta nas águas naturais devido às descargas de efluentes industriais, principalmente as galvanoplastias, produção de pigmentos, soldas, equipamentos eletrônicos, lubrificantes e acessórios fotográficos. É também usado na formulação de produtos inseticidas. A queima de combustíveis fósseis consiste também numa fonte de cádmio para o ambiente (CETESB, 2007). Os efeitos prejudiciais do cádmio na saúde incluem uma série de desordens crônicas, como a doença de itai-itai, danos renais, enfisemas, hipertensão e atrofia testicular (LEYVA-RAMOS et al., 1997).

Diante da crescente geração de efluentes, faz-se necessária a busca de alternativas que sejam eficientes tecnicamente, economicamente e que ao mesmo tempo sejam menos poluentes (QUINTELAS et al., 2008). Vários métodos vêm sendo utilizados para remoção de íons metálicos de soluções, tais como precipitação química (JANEGITZ et al., 2007; MATLOCK et al., 2002), troca iônica (DABROWSKI et al., 2004), adsorção (ANDRABI, 2011; DETCHEVA et al., 2011, MOHAMMAD et al., 2010), tratamento eletroquímico (HUNSOM et al., 2005), mas nem sempre são eficientes e apresentam-se com elevado custo (DAS et al., 2008), principalmente quando aplicados a soluções diluídas de metais (QUINTELAS et al., 2008). Portanto, a busca de novas tecnologias de tratamento desses efluentes que apresente vantagens em relação ao custo-benefício do processo vem direcionando ao aumento no interesse sobre o processo de biossorção (DAS et al., 2008).

A biossorção consiste na absorção de metais tóxicos por microrganismos (biomassa) vivos ou mortos (QUINTELAS et al., 2008). De acordo com Pietrobelli et al. (2009), a biossorção é uma alternativa eficaz aos tratamentos de efluentes convencionais já utilizados, pois, os microrganismos retém os metais promovendo uma auto-regeneração do efluente e qualificando o processo. Assim, o processo da biossorção surge como uma tecnologia promissora e em atual expansão em relação à sua aplicação no tratamento e polimento final de efluentes contendo metais tóxicos (MÓDENES et al., 2009; KRATOCHVIL et al., 1998).

Diferentes materiais biológicos vêm sendo empregados visando a biossorção de metais tóxicos, tais como biomassas de bactérias (WIERZBA, 2010; CHEN et al., 2009; MESQUITA et al., 1998), fungos (AKAR E TUNALI et al., 2006; FRANCO et al., 2004), algas (SARAVANAN et al., 2009; CRUZ et al., 2004; COSTA et al., 1994) e macrófitas aquáticas (LIMA et al., 2011).

Os biossurfactantes são moléculas tensoativas produzidas por microrganismos que apresentam aplicações em biorremediação (NITSCHKE e PASTORE, 2002) podendo auxiliar também nos processos de remoção de metais pesados de meios líquidos por microrganimos (MULLIGAN et al., 2001). Objetivou-se a remoção de cádmio por fungos filamentosos em bioprocesso submerso e a concomitante produção de biossurfactantes. 


\section{MATERIAIS E MÉTODOS}

\subsection{Microrganismos}

Os microrganismos utilizados foram fungos filamentosos do gênero Aspergillus ssp identificados por O-3 e O-8 e do gênero Trichoderma sp identificado como E-19. Os fungos identificados com a inicial "O" foram isolados de solo contaminado com óleo diesel e os com inicial "E” foram isolados de efluentes de laticínios por COLLA et al. (2009).

\subsection{Manutenção do microrganismo e preparo do inóculo}

Os microrganismos foram mantidos em laboratório cultivados em ágar-batata-destrose (ABD) em placas de Petri e refrigerados a $4^{\circ} \mathrm{C}$. Para a ativação dos fungos, estes foram repicados para placas de Petri com $30 \mathrm{~mL}$ de meio de cultivo $\mathrm{ABD}$ e incubados a $30^{\circ} \mathrm{C}$ durante $7 \mathrm{~d}$. Os esporos fúngicos foram utilizados para a inoculação dos meios de cultivo.

\subsection{Preparo do meio de cultivo líquido contendo cádmio}

O meio de cultivo foi composto por $10 \%$ de farelo de trigo, $10 \%$ de solução salina, $4,5 \%$ de extrato de levedura como fonte de nitrogênio, $2 \%$ de óleo de soja como fonte de carbono adicional e $10 \mathrm{mg} / \mathrm{L}$ ou $20 \mathrm{mg} / \mathrm{L}$ de cádmio. A solução salina objetiva a adição de micronutrientes, sendo composta por $\mathrm{KH}_{2} \mathrm{PO}_{4}-2 \mathrm{~g} / \mathrm{L} ; \mathrm{MgSO}_{4}-1 \mathrm{~g} / \mathrm{L}$ e solução traço $-10 \mathrm{~mL} / \mathrm{L}$, sendo a solução traço composta por $\mathrm{FeSO}_{4} \cdot 7 \mathrm{H}_{2} \mathrm{O}-0,63 \mathrm{mg} ; \mathrm{MnSO}_{4}-0,01 \mathrm{mg} ; \mathrm{ZnSO}_{4}-0,62 \mathrm{mg}$ e água destilada ate o volume de $1 \mathrm{~L}$. O óleo de soja foi adicionado a fim de estimular a produção de biossurfactantes pelos microrganismos.

O meio foi preparado pelo cozimento do farelo de trigo a $100^{\circ} \mathrm{C}$ em água destilada durante 30 min, seguido de filtração. Ao filtrado foi adicionada a solução salina, as fontes de nitrogênio e carbono e a solução padrão de cádmio, além da água destilada necessária para completar o volume final do meio. $\mathrm{O} \mathrm{pH}$ do meio foi ajustado para 7,0 através da adição de $\mathrm{H}_{2} \mathrm{SO}_{4}(1,5 \mathrm{~mol} / \mathrm{L})$ e autoclavado. $\mathrm{O}$ meio estéril foi distribuído em erlenmeiers de $300 \mathrm{~mL}$ estéreis em um volume inicial de meio de $100 \mathrm{~mL}$.

\subsection{Inoculação e incubação}

A inoculação dos meios foi realizada utilizando áreas circulares de $2 \mathrm{~cm}$ de diâmetro do crescimento fúngico das placas de Petri contendo o meio ABD. Os erlenmeiers contendo o meio inoculado foram incubados a $30^{\circ} \mathrm{C}$ durante $7 \mathrm{~d}$ em agitador orbital, com agitação de $120 \mathrm{rpm}$.

Alíquotas foram coletadas nos tempos inicial e final do bioprocesso para a determinação da concentração de cádmio presente e avaliação do processo de remoção. Também foram realizadas coletas de amostras nos tempos $0,1,2,3,5$ e 7 d para extração do biossurfactante.

\subsection{Delineamento experimental}

Foram realizados experimentos com 3 fungos filamentosos Aspergillus cepas O-3 e O-8 e Thrichoderma cepa E-19 e duas concentrações iniciais de cádmio no meio (10 e $20 \mathrm{mg} / \mathrm{L})$. Os experimentos foram realizados em duplicata, totalizando 12 experimentos. 


\subsection{Determinação da absorção do metal}

As amostras para análises da absorção do metal foram digeridas pelo Método 30 50B (U. S. Environmental Protection Agency) e as concentrações determinadas através de absorção atômica (espectrofotômetro Analyst 200-Perkin Elmer), no Laboratório de Solos da Universidade de Passo Fundo.

\subsection{Extração do biossurfactante e determinação da atividade emulsificante}

As alíquotas retiradas periodicamente de cada experimento foram centrifugadas durante 30 min a $4000 \mathrm{rpm}$ para remoção da biomassa fúngica e o sobrenadante obtido utilizado como extrato para a determinação da atividade emulsificante dos meios. A atividade emulsificante (AE) óleo em água (O/A) dos extratos foi determinada segundo metodologia proposta por Martins et al. (2007), sendo calculada através da Equação 1. Em tubos de ensaio adicionou-se $3,5 \mathrm{~mL}$ do meio de fermentação livre de células diluído $(\mathrm{D}=10)$ 1:10 e $2 \mathrm{~mL}$ de óleo de milho. A mistura foi agitada em agitador Vórtex a $700 \mathrm{rpm}$ por $1 \mathrm{~min}$. Após $60 \mathrm{~min}$ de repouso foi lida a absorbância do meio emulsificado óleo em água em espectrofotômetro a $610 \mathrm{~nm}$. Um branco foi realizado como controle, utilizando água ao invés do meio de fermentação. Os resultados foram expressos em EU (unidades de emulsificação).

$$
\mathrm{AE}_{\mathrm{O} / \mathrm{A}}=\left(\mathrm{ABS}_{\text {amostra }}-\mathrm{ABS}_{\text {branco }}\right) \cdot \mathrm{D}
$$

Os resultados de atividade emulsificante foram submetidos à Análise de Variância e Teste de Tukey para comparação de médias a 5\% de significância.

\section{RESULTADOS E DISCUSSÕES}

A Tabela 1 apresenta a concentração de $\mathrm{Cd}(\mathrm{mg} / \mathrm{L})$ presente nos meios da fermentação submersa com os fungos E-19, O-8 e O-3, determinada através de absorção atômica, nos tempos inicial e final de fermentação.

Tabela 1: Absorção de cádmio em função do tempo

\begin{tabular}{cccc}
\hline & \multicolumn{2}{c}{ Concentração Cd (mg/L) } & \multirow{2}{*}{$\begin{array}{c}\text { Remoção de } \\
\text { Cúdmio }(\%)\end{array}$} \\
\cline { 2 - 3 } Fungos & Inicial & Final & 46 \\
E-19 & 10,1 & 5,3 & 49 \\
& 20,7 & 10,0 & 100 \\
O-8 & 10,1 & ND & 100 \\
& 20,7 & ND & 100 \\
O-3 & 10,1 & ND & 100 \\
& 20,7 & ND &
\end{tabular}


Os experimentos realizados com o fungo Trichoderma E-19 apresentaram concentrações de cádmio no meio de cultivo ao final da fermentação acima dos limites estabelecidos pela Resolução Conama $\mathrm{n}^{\circ} 357 / 05$, que é de $0,001 \mathrm{mg} / \mathrm{L}$, sendo as remoções finais de $46 \%$ e $49 \%$ para as concentrações de $10 \mathrm{mg} / \mathrm{L}$ e $20 \mathrm{mg} / \mathrm{L}$, respectivamente. Já os fungos filamentosos O-8 e O-3, ambos do gênero Aspergillus apresentaram as maiores remoções de cádmio, de $100 \%$, independente das concentrações iniciais de cádmio utilizadas $(10 \mathrm{mg} / \mathrm{L}$ ou $20 \mathrm{mg} / \mathrm{L})$. A remoção $\mathrm{Cd}$ por espécies de Aspergillus é relatada com freqüência na literatura. Por exemplo, Al-Garni et al. (2009) estudaram a biossorção $\mathrm{Cd}$ de meios de cultivo por 19 espécies fúngicas, sendo que uma cepa de Aspergillus fumigatus apresentou potencial para sobrevivência em meios contendo até $1 \mathrm{mg} / \mathrm{mL}$ de cádmio. $\mathrm{O}$ microrganismo inativado através de métodos químicos também apresentou potencial de biossorção de Cd. Sun et al. (2010) citaram a remoção de $\mathrm{Pb}+2, \mathrm{Hg}+2$ e $\mathrm{Cd}+2$ por células de Aspergillus terreus imobilizadas em suportes.

A Tabela 2 apresenta as atividades emulsificantes óleo em água $\left(\mathrm{AE}_{\mathrm{O} / \mathrm{A}}\right)(\mathrm{UE})$ em função do tempo de cultivo (d), obtidas nos cultivos submersos com fungos filamentosos na presença $10 \mathrm{mg} / \mathrm{L} \mathrm{e}$ $20 \mathrm{mg} / \mathrm{L}$ de cádmio. A análise de variância dos resultados de atividade emulsificante está apresentada na Tabela 3.

Tabela 2: Atividades Emulsificantes óleo em água (UE) ao longo do tempo de fermentação e condições experimentais

\begin{tabular}{ccccccc}
\hline Fungo & \multicolumn{2}{c}{ Thrichoderma $\mathrm{E}-19$} & \multicolumn{2}{c}{ Aspergillus $\mathrm{O}-8$} & \multicolumn{2}{c}{ Aspergillus O-3 } \\
\hline$[\mathrm{Cd}]_{0}(\mathrm{mg} / \mathrm{L})$ & 10 & 20 & 10 & 20 & 10 & 20 \\
\hline $0 \mathrm{~d}$ & $1,56 \pm 0,53^{\text {efgh }}$ & $1,45 \pm 0,32^{\text {fgh }}$ & $1,56 \pm 0,53^{\text {efgh }}$ & $1,45 \pm 0,32^{\text {fgh }}$ & $1,56 \pm 0,53^{\text {efgh }}$ & $1,45 \pm 0,32^{\text {fgh }}$ \\
$1 \mathrm{~d}$ & $1,11 \pm 0,81^{\text {gh }}$ & $3,26 \pm 0,0^{\text {defg }}$ & $2,28 \pm 0,15^{\text {efgh }}$ & $0,13 \pm 0,04^{\text {h }}$ & $2,45 \pm 1,12^{\text {efg }}$ & $2,92 \pm 0,29^{\text {defg }}$ \\
$2 \mathrm{~d}$ & $2,26 \pm 1,06^{\text {efgh }}$ & $3,59 \pm 0,34^{\text {cdef }}$ & $7,98 \pm 0,82^{\text {a }}$ & $7,48 \pm 0,23^{\text {ab }}$ & $2,04 \pm 0,66^{\text {efgh }}$ & $2,87 \pm 0,04^{\text {defg }}$ \\
$3 \mathrm{~d}$ & $1,44 \pm 0,75^{\text {fgh }}$ & $1,51 \pm 0,52^{\text {gh }}$ & $7,21 \pm 1,15^{\text {ab }}$ & $2,38 \pm 0,63^{\text {efg }}$ & $1,76 \pm 0,33^{\text {efgh }}$ & $1,15 \pm 0,31^{\text {gh }}$ \\
$5 \mathrm{~d}$ & $1,24 \pm 0,26^{\text {fgh }}$ & $2,42 \pm 0,17^{\text {efg }}$ & $1,57 \pm 0,56^{\text {efgh }}$ & $2,17 \pm 0,09^{\text {efgh }}$ & $2,66 \pm 0,26^{\text {efg }}$ & $1,06 \pm 1,28^{\text {hg }}$ \\
$7 \mathrm{~d}$ & $1,72 \pm 0,18^{\text {efgh }}$ & $2,69 \pm 0,81^{\text {defg }}$ & $3,74 \pm 0,12^{\text {cde }}$ & $5,54 \pm 0,41^{\text {bc }}$ & $3,14 \pm 0,59^{\text {defg }}$ & $4,76 \pm 0,15^{\text {cd }}$ \\
\hline
\end{tabular}

* Resultados de média \pm desvio padrão. Médias seguidas de letras diferentes apresentam diferenças significativas a um nível de significância de $5 \%$. $[\mathrm{Cd}]_{0}=$ concentração inicial de cádmio nos cultivos

Tabela 3: Análise de variância dos resultados de Atividade Emulsificante O/A

\begin{tabular}{cccccc}
\hline Fonte de variação & $\begin{array}{c}\text { Soma dos } \\
\text { Quadrados }\end{array}$ & $\begin{array}{c}\text { Graus de } \\
\text { Liberdade }\end{array}$ & $\begin{array}{c}\text { Quadrado } \\
\text { Médio }\end{array}$ & Valor F & $\begin{array}{c}\text { Nívelde } \\
\text { significância }(p)\end{array}$ \\
\hline Média & 497,4532 & 1 & 497,4532 & 1807,193 & 0,000000 \\
Fungo $\left(\mathrm{X}_{1}\right)$ & 32,5236 & 2 & 16,2618 & 59,077 & 0,000000 \\
{$[\mathrm{Cd}]$ inicial $(\mathrm{mg} / \mathrm{L})\left(\mathrm{X}_{2}\right)$} & 0,7852 & 1 & 0,7852 & 2,853 & 0,100120 \\
Tempo $(\mathrm{d})\left(\mathrm{X}_{3}\right)$ & 74,9822 & 5 & 14,9964 & 54,480 & 0,000000 \\
$\mathrm{X}_{1} \cdot \mathrm{X}_{2}$ & 13,8780 & 2 & 6,9390 & 25,209 & 0,000000 \\
$\mathrm{X}_{1} \cdot \mathrm{X}_{3}$ & 74,9152 & 10 & 7,4915 & 27,216 & 0,000000 \\
$\mathrm{X}_{2} \cdot \mathrm{X}_{3}$ & 19,1338 & 5 & 3,8268 & 13,902 & 0,000000 \\
$\mathrm{X}_{1} \cdot \mathrm{X}_{2} \mathrm{X}_{3}$ & 14,9897 & 10 & 1,4990 & 5,446 & 0,000077 \\
Erro & 9,6342 & 35 & 0,2753 & & \\
\hline
\end{tabular}

Verificou-se que as variáveis fungo e tempo de cultivo foram significativas $(\mathrm{p}<0,05$, Tab.3) sobre a atividade emulsificante O/A obtida nos cultivos submersos. Como a interação de terceira ordem entre as variáveis foi significativa, esta foi considerada para a realização do teste de Tukey a $5 \%$ de significância, obtendo-se as comparações entre médias mostradas na Tabela 2.

As médias de atividade emulsificante para as condições experimentais testadas foram apresentadas na Figura 1, verificando-se que as maiores atividades emulsificantes (7,2 a 7,9 UE) foram obtidas pelo fungo Aspergillus O-8, entre o segundo e terceiro dias de fermentação, independente da concentração inicial de Cd presente no meio de cultivo. 
A atividade emulsificante é um indício da produção de biossurfactantes pelos microrganismos, uma vez que estes compostos, por serem constituídos de porções polares e apolares nas moléculas, são capazes de ocasionar a emulsificação de sistemas bifásicos constituídos por óleo e água, como é o caso das emulsões formadas a partir do teste de emulsificação. Comparando-se os resultados de atividade emulsificante com os resultados de remoção de metais, verifica-se que este fungo apresentou $100 \%$ de remoção de $\mathrm{Cd}$ do meio de fermentação.

Segundo Miller et al. (1993) os biossurfactantes apresentam potencial para aumentar as eficiências de remoção de metais em solos por facilitarem processos de sorção, transferência de massa e resistência ao transporte em fase aquosa. A adição de biossurfactantes pode promover a desorção de metais pesados de fases sólidas através da complexação das formas livres dos metais em solução ou pela acumulação dos biossurfactantes na interface líquido-sólido causada pela redução das atividades interfaciais, o que promove um contato direto entre os biossurfactantes e os metais. Ainda, Valdman et al. (2005) demonstraram que um exopolissacarídio extraído do microrganismo Serratia sp, com atividade biossurfactante, apresentou elevada eficiência na remoção de $\mathrm{Cd}$ de meios líquidos. Os experimentos foram realizados com $50 \mathrm{mg} / \mathrm{L} \mathrm{de} \mathrm{Cd}^{2+}$ e $0.1 \mathrm{~g} / \mathrm{L}$ do biossurfactante previamente purificado, obtendo-se remoções da ordem de $170 \mathrm{mg}$ de $\mathrm{Cd} / \mathrm{g}$ de biossurfactante.

Entretanto, verificou-se que o fungo Aspergillus O-3, embora não tenha apresentado elevadas atividades emulsificantes, também apresentou remoções totais do metal dos meios de cultivo. Isto pode ser justificado pelo de determinados biossurfactantes serem produzidos no meio intracelular sem serem excretados para o meio.
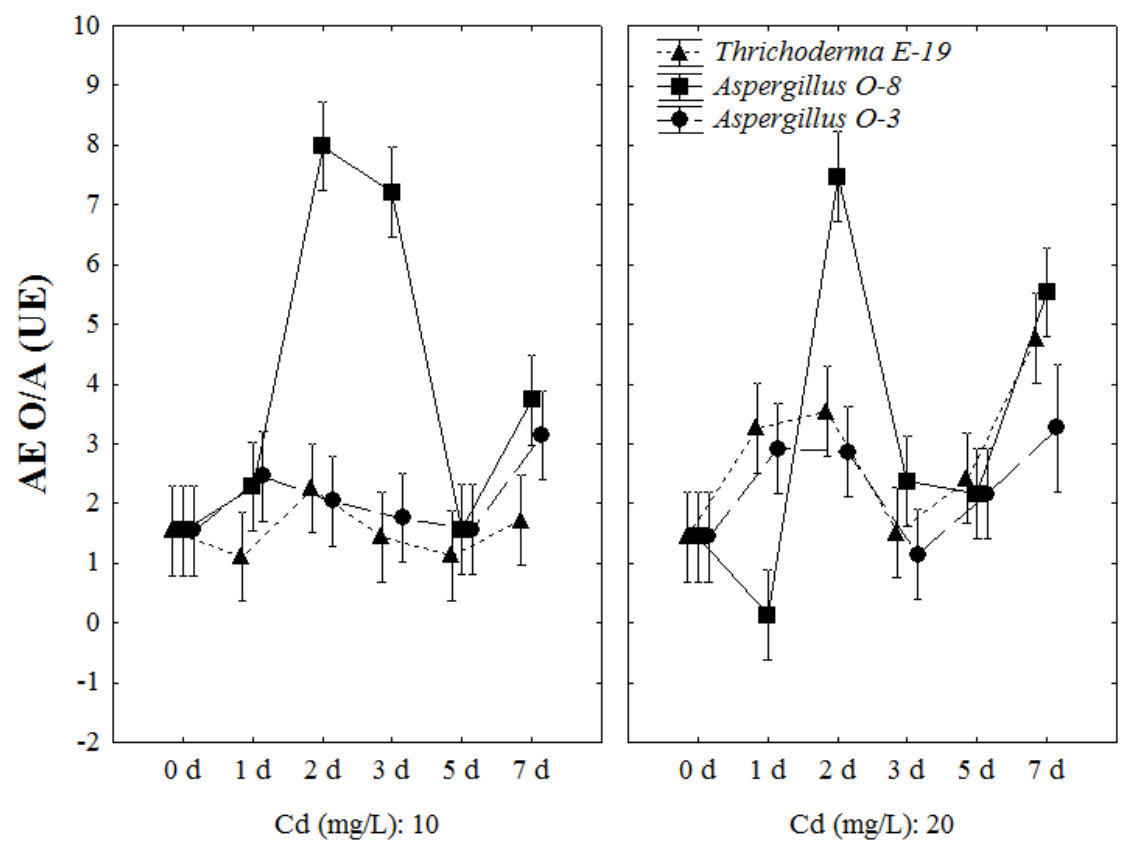

Figura 1: Atividade emulsificante $\mathrm{O} / \mathrm{A}$ (UE) versus tempo de cultivo nos meios com concentrações iniciais de cádmio de (a) $10 \mathrm{mg} / \mathrm{L}$ e (b) $20 \mathrm{mg} / \mathrm{L}$. $\mathbf{\Delta}$ fungo E-19; • fungo O-8; • fungo O-3.

\section{CONCLUSÃO}

Este trabalho apresenta dados importantes acerca da capacidade de fungos filamentosos para a remoção de $\mathrm{Cd}$ de meios líquidos. Dos gêneros estudados, espécies de Aspergillus apresentaram maiores remoções de $\mathrm{Cd}(100 \%)$ do que uma espécie de Thrichoderma (O-3 e O-8). Durante os processos de biossorção, foi observada a produção de biossurfactantes pelos microrganismos 
estudados, demonstrando a importância destes compostos nos processos de remoção de metais de meios líquidos.

\section{REFERÊNCIAS BIBLIOGRÁFICAS}

AKAR, T.; TUNALI, S. Biosorption characteristics of Aspergillus flavus biomass for removal of $\mathrm{Pb}$ (II) and $\mathrm{Cu}$ (II) ions from an aqueous solution. Bioresource Technology, v. 97, n. 15, p. 1780-1787, 2006.

AL-GARNI, S.M.; GHANEM, K.M.; BAHOBAIL, A. Biosorption characteristics of Aspergillus fumigatus in removal of cadmium from an aqueous solution. African Journal of Biotechnology, v. 8, n. 17 , p. 4163-4172, 2009.

ANDRABI, S. M. A. Sawdust of lam tree (Cordia africana) as a low-cost, sustainable and easily available adsorbent for the removal of toxic metals like $\mathrm{Pb}$ (II) and $\mathrm{Ni}$ (II) from aqueous solution.

European Journal of Wood and Wood Products, v. 69, p. 75-83, 2011.

CETESB, Companhia de Tecnologia de Saneamento Ambiental de São Paulo. Anexo 8: Monitoramento da qualidade das águas de rios litorâneos. Disponível em: <http://www.cetesb.sp.gov.br/Agua/rios/publicacoes.asp>. Acesso em: 03 abr. 2009.

CHEN, X.; HU, S.; SHEN, C.; DOU, C.; SHI, J.; CHEN, Y. Interaction of Pseudomonas putida CZ1 with clays and ability of the composite to immobilize copper and zinc from solution. Bioresource Technology, v. 100, n. 1, p. 330-337, 2009.

COLLA, L. M. et al., A Solid-State Bioprocess for Selecting Lipase-Producing Filamentous Fungi. Z. Naturforsch, v. 64c, p. 131-137, 2009.

CONAMA Resolução 357/2005 $\quad \mathrm{n}^{\circ} \quad$ 128/2006. Disponível em: <http://www.mma.gov.br/sitio/index.php?ido=legislacao.index\&tipo=0>. Acesso em 14 jun. 2009.

COSTA, A.C.A.; TELES, E.M.F.; LEITE, S.G.F. Accumulation of cadmium from moderately concentrated cadmium solutions by Chlorella and Scenedesmus strains. Journal of Microbiology, v. 25, p. 42-45, 1994.

CRUZ, C.C.V.; DA COSTA, A. C. A.; HENRIQUES, C. A.; LUNA, A. S. Kinetic modeling and equilibrium studies during cadmium biosorption by dead Sargassum sp. Biomass. Bioresource Technology, v. 91, n. 33, p. 249-257, 2004.

DABROWSKI, A.; HUBICKI, Z.; PODKOSCIELNY, P.; ROBENS, E. Selective removal of the heavy metal ions from waters and industrial wastewaters by ion-exchange method. Chemosphere, $v$. 56, n. 2, p. 91-106, 2004.

DAS, N.; VIMALA, R.; KARTHIKA, P. Biosorption of heavy metals - An Overview. Indian Journal of Biotechnology, v. 7, p. 159-169, 2008.

DETCHEVA, A.K., VASSILEVA, P.S., GEORGIEVA, R.H., VOYKOVA, D.K., GERGANOVA, T.I. AND IVANOVA, Y.Y. Adsorption properties of a nanostructured hybrid material containing 
aluminium towards some metal ions. Central European Journal of Chemistry, v. 9, n. 5, p. 932-940 2011.

FARIAS, Y. P. M. M., Aplicação de microrganismos na remediação de áreas contaminadas por metais pesados. XVI Jornada de Iniciação Cientifica, CETEM, 2008.

FRANCO, L.O.; MAIA, R. C. C.; PORTO, A. L. F.; MESSIAS, A. S.; FUKUSHIMA, K.; CAMPOSTAKAKI, G. M. C. Heavy metal biosorption by chitin and chitosan isolated from Cunninghamella elegans (IFM 46109). Brazilian Journal of Microbiology, v.35, p. 243-247. 2004.

FREITAS, T. C. M.; MELNIKOV, P. O uso e os impactos da reciclagem de cromo em indústrias de curtume em Mato Grosso do Sul, Brasil. Engenharia Sanitária e Ambiental, v. 11, n. 4, p. 305-310, 2004.

GAAD, G. M. Biosorption. Chemistry and industry, v.2, p. 421-426, 1990.

HUNSOM, M.; PRUKSATHORN, K.; DAMRONGLERD, S.; VERGNES, H.; DUVERNEUIL, P. Electrochemical treatment of heavy metals $\left(\mathrm{Cu}^{2+}, \mathrm{Cr}^{6+}, \mathrm{Ni}^{2+}\right)$ from industrial effluent and modeling of copper reduction. Water Research, v. 39, n. 4, p. 610-616, 2005.

JANEGITZ, B.C.; LOURENÇÃO, B.C.; LUPETTI, K.O.; FATIBELLO-FILHO, O. Desenvolvimento de um método empregando quitosana para remoção de íons metálicos de águas residuárias. Química Nova, v. 30, n. 4, p. 879-884, 2007.

JIMENEZ, R. S.; DAL BOSCO, S. M.; CARVALHO, W. A. Remoção de metais pesados de efluentes aquosos pela zeólita natural escolecita - influência da temperatura e do $\mathrm{pH}$ na adsorção em sistemas monoelementares. Química Nova, v. 27, n. 5, p. 734-738, 2004.

KORF, E. P.; MELO, E. F. R. Q.; THOMÉ, A.; ESCOSTEGUY, P. A. V. Retenção de metais em solo da antiga área de disposição de resíduos sólidos urbanos de Passo Fundo - RS. Revista de Ciências Ambientais, v. 2, n. 2, p. 43-60, 2008.

KRATOCHVIL, D.; VOLESKY, B. Advances in the Biosorption of Heavy Metals. Tibtech, v. 16, p. 291-300, 1998.

Leyva-Ramos, R; Rangel-Mendez, J. R.; Mendoza-Barron, J.; Fuentes-Rubio, L.; GuerreroCoronado, R. M.Adsorption of cadmium(II) from aqueous solution on activated carbon. Water Sci. Technol., v.35, n. 7, p. 205-211, 1997.

LIMA, L. K. S.; KLEINÜBING, S. J.; EDSON ANTONIO DA SILVA, E. A.; DA SILVA, M. G. C. Removal of Chromium from Wastewater Using Macrophytes Lemna Minor as Biosorbent. Chemical Engineering Transactions, v. 25, p. 303-308, 2011.

MARTINS, V.G.; KALIL, S.J.; COSTA, J.A.V. Co-produção de lipase e biossurfactante em estado sólido para utilização em biorremediação de óleos vegetais e hidrocarbonetos. Química Nova, v.31, n.8, p. 1942-1947, 2008.

MATLOCK, M. M.; HOWERTON, B. S.; ATWOOD, D. A. Chemical precipitation of heavy metals from acid mine drainage. Water Research, v. 36, n. 19, p. 4757-4764, 2002. 
MERLINO, L. C. S.; MELO, W. J.; MACEDO, F. G.; GUEDES, A. C. T. P.; RIBEIRO, M. H.; MELO, V. P.; MELO, G. M. P. Bário, Cádmio, Cromo e Chumbo em plantas de milho e em latossolo após onze aplicações anuais de lodo de esgoto. Revista Brasileira de Ciência do Solo, v. 34, p. 20312039, 2010.

MESQUITA, L.M.S.; GONÇALVES, M.M.M.; LEITE, S.G.F. Influence of the maintenance method on the cadmium biosorption capacity of Micrococcus luteus. Journal of Microbiology, v. 29, p. 4043, 1998.

MILLER, R.M. Biosurfactant-facilitated remediation of metal-contaminated soils. Environ Health Perspect., v. 103, n. 1, p. 59-62, 1995.

MÓDENES, A. N.; PIETROBELLI, J. M. T. A.; QUIÑONES, F. R. E.; SUZAKI, P. Y. R.; ALFLEN, V. L.; KLEN, M. R. S. F. Potencial de biossorção do zinco pela macrófita Egeria densa. Engenharia Sanitária e Ambiental, v. 14, n. 4, p. 465-470, 2009.

MOHAMMAD, M.; MAITRA, S.; AHMAD, N.; BUSTAM, A.; SEN, T. K.; DUTTA, B. K. Metal ion removal from aqueous solution using physic seed hull. Journal of Hazardous Materials, v. 179, n. 1-3, p. 363-372, 2010.

MULLIGAN, C. N.; YONG, R. N.; GIBES, B. F. Heavy metal removal from sediments by biosurfactants. Journal Hazardous Materials, v. 85, p 11-15, 2001.

MULLIGAN, C. N.; YONG, R. N.; GIBES, B. F. Metal removal from contamined soil and sediments by biosurfactants surfaction. Environmental Science and Technology. v. 33, p. 3812-3820, 1999.

MULLIGAN, C. N.; YONG, R. N.;GIBBS, B. F. Surfactant-enhanced remediation of contaminated soil: a review. Engineering Geology, v. 60, p. 371-380, 2001.

MURALEEDHARAN, T.R.; IYENGAR, L.; VENKOBACHAR, C. Biosorption: an attractive alternative for metal removal and recovery. Current Science, v. 61, n. 6, p. 379-385, 1991.

NITSCHKE, M.; PASTORE, G.M. Biossurfactantes: propriedades e aplicações. Química Nova, v. 25, n. 5, p. 772-776, 2002.

PANDEY, A.; SOCCOL, C. R.; MITCHELL, D. New developments in solid state fermentation: Ibioprocesses and products. Process Biochemistry, v.35, p. 1153-1169, 2000.

PIETROBELli, J. M. T. A.; MÓDENES, A. N.; KLEN, M. R. S. F.; QUIÑONES, F. R. E. Cadmium, copper and zinc biosorption study by non-living Egeria densa biomass. Water Air Soil Pollution, v. 202, p. 385-392, 2009.

QUINTELAS, C.; FERNANDES, B.; CASTRO, J.; FIGUEIREDO, H.; TAVARES, T. Biosorption of $\mathrm{Cr}(\mathrm{VI})$ by three different bacterial species supported on granular activated carbon - A comparative study. Journal of Hazardous Materials, v. 153, p. 799-809, 2008.

REGULY, J.C. Biotecnologia dos processos fermentativos: fundamentos, matérias-primas agrícolas, produtos e processos. Pelotas, RS, v 3. 1996. 
SARAVANAN, A.; BRINDHA, V.; MANIMEKALAI, R.; KRISHNAN, S. An evaluation of chromium and zinc biosorption by a sea weed (Sargassum sp.) under optimized conditions. Indian Journal of Science and Technology, v.2, n.1, p. 53-56, 2009.

SCHNEIDER, I.A.H.; RUBIO, J.; SMITH, R.W. Biosorption of metals onto plant biomass: exchange adsorption or surface precipitation? International Journal of Mineral Processing, v. 62, p. 111-120, 2011.

SILVA, E. P.et al. Biosorção de Cádmio pelo Aspergillus Níger. $2^{\circ}$ Congresso Brasileiro de P\&D Petróleo e Gás. Universidade Federal do Rio Grande do Norte - UFRN, Natal/RN - Brasil.

SUN, Y.M.; HORNG, C.Y.; CHANG, F.L.; CHENG, L.C.; TIAN, W.X. Biosorption of lead, mercury, and cadmium ions by Aspergillus terreus immolilized in a natural matriz. Polish Journal of Microbiology, v. 59, n. 1, p. 37-44, 2010.

VALDMAN, E.; LEITE, S.G.; CALDAS, S.M. Effect of biosurfactant concentration on cadmium biosorption by Serratia sp isolated from tropical soil. In: Proceedings of XIII International Conference on Heavy Metals in the Environment, Rio de Janeiro, Brasil, 2005.

WIERZBA, S. Heavy metals biosorption from aqueous solution by Pseudomonas sp. G1. Proceedings of ECOpole, v. 4, n. 1, p. 85-89, 2010. 